\title{
ON A DIOPHANTINE EQUATION ON TRIANGULAR NUMBERS
}

\author{
ABDELKADER HAMTAT AND DJILALI BEHLOUL
}

Received 06 February, 2015

\begin{abstract}
A number $N$ is a triangular number if it can be written as $N=0+1+\cdots+n$ for some natural number $n$. We study the problem of finding all nonnegative integer solutions of the Diophantine equation $(0+1+\cdots+X)+(0+1+\cdots+Y)=(0+1+\cdots+Z)$. Using this equation, some new and curious increasing integer sequences are built.
\end{abstract}

2010 Mathematics Subject Classification: 11D25; 11D41

Keywords: Diophantine equation, triangular numbers

\section{INTRODUCTION}

A triangular number is a number of the form $T_{n}=\sum_{k=0}^{n} k=n(n+1) / 2$, where $n$ is a natural number.

So the first few triangular numbers are $0,1,3,6,10,15,21,28, \ldots$ (sequence A000217 in [6]). A well known fact about the triangular numbers is that $X$ is a triangular number if and only if $8 X+1$ is a perfect square. Triangular numbers can be thought of as the numbers of dots needed to make a triangle.

We are concerned by the Diophantine equation of the form

$$
T_{X}+T_{Y}=T_{Z}
$$

Papers [1-5] gave many interesting results concerning the problem of solvability of Diophantine equations related to triangular numbers. The aim of this paper is twofold: on the one hand, it gives all solutions of the Diophantine equation (1.1), and on the other hand, it gives us a method of finding explicitly (and quickly) infinite families of solutions of (1.1) in where many new integer sequences are derived.

\section{General SOLUTiON}

Let us start with some technical lemmas.

Lemma 1. Let $a, b, c, d \in \mathbb{N}$. Then $a b=c d$ if and only if there exist $p, q, m, n \in \mathbb{N}$ such that $a=m n, b=p q, c=m p$ and $d=n q$. 
Proof. Let $a=\prod_{i \in A \subset \mathbb{N}} p_{i}^{\alpha_{i}}$ and $b=\prod_{i \in B \subset \mathbb{N}} q_{i}^{\beta_{i}}$ where $p_{i}, q_{i}$ are primes, $\alpha_{i}, \beta_{i} \in$ $\mathbb{N}$ and $A, B$ are finite sets. The fact $c d=\prod_{i \in A \subset \mathbb{N}} p_{i}^{\alpha_{i}} \prod_{i \in B \subset \mathbb{N}} q_{i}^{\beta_{i}}$ implies that $c=$ $\prod_{i \in A^{\prime} \subset A} p_{i}^{\alpha_{i}^{\prime}} \prod_{i \in B^{\prime} \subset B} q_{i}^{\beta_{i}^{\prime}}$ and $d=\prod_{i \in A^{\prime \prime} \subset A} p_{i}^{\alpha_{i}^{\prime \prime}} \prod_{i \in B^{\prime \prime} \subset B} q_{i}^{\beta_{i}^{\prime \prime}}$, where $0 \leq \alpha_{i}^{\prime}, \alpha_{i}^{\prime \prime} \leq \alpha_{i}, 0 \leq$ $\beta_{i}^{\prime}, \beta_{i}^{\prime \prime} \leq \beta_{i}$ with $\alpha_{i}=\alpha_{i}^{\prime}+\alpha_{i}^{\prime \prime}$ and $\beta_{i}=\beta_{i}^{\prime}+\beta_{i}^{\prime \prime}$. If we put $m=\prod_{i \in A^{\prime} \subset A} p_{i}^{\alpha_{i}^{\prime}}, p=$ $\prod_{i \in B^{\prime} \subset B} q_{i}^{\beta_{i}^{\prime}}, n=\prod_{i \in A^{\prime \prime} \subset A} p_{i}^{\alpha_{i}^{\prime \prime}}$ and $q=\prod_{i \in B^{\prime \prime} \subset B} q_{i}^{\beta_{i}^{\prime \prime}}$ then we obtain, $a=m n, b=$ $p q, c=m p$ and $d=n q$.

Lemma 2. Equation (1.1) is equivalent to $X(X+1)=(Z-Y)(Z+Y+1)$.

Proof. Replacing $T_{X}$ by $\frac{X(X+1)}{2}, T_{Y}$ by $\frac{Y(Y+1)}{2}$ and $T_{Z}$ by $\frac{Z(Z+1)}{2}$ equation (1.1) becomes

$$
\frac{X(X+1)}{2}+\frac{Y(Y+1)}{2}=\frac{Z(Z+1)}{2},
$$

i.e.,

$$
X^{2}+X+Y^{2}+Y=Z^{2}+Z \text {. }
$$

Thus

$$
X(X+1)=(Z-Y)(Z+Y+1) .
$$

Now, we are able to establish the following theorem.

Theorem 1. All nonnegative integer solutions of the equation $T_{X}+T_{Y}=T_{Z}$ are

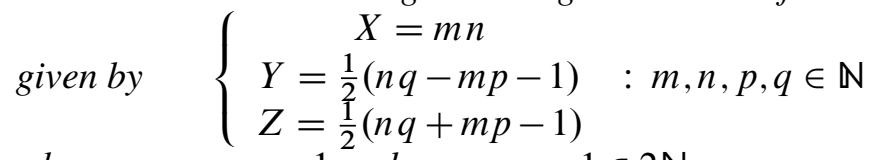
where $p q-m n=1$ and $n q-m p-1 \in 2 \mathbb{N}$.

Proof. According to Lemma 2, (1.1) is equivalent to $X(X+1)=(Z-Y)(Z+$ $Y+1)$, thus thanks to Lemma 1, one has

$$
\begin{aligned}
X & =m n \\
X+1 & =p q \\
Z-Y & =m p \\
Z+Y+1 & =n q \text { where } m, n, p, q \in \mathbb{N} .
\end{aligned}
$$

Therefore we get

$$
\begin{aligned}
& X=m n \\
& Y=\frac{1}{2}(n q-m p-1)
\end{aligned}
$$




$$
Z=\frac{1}{2}(n q+m p-1) \text { where } m, n, p, q \in \mathbb{N}
$$

such that $p q-m n=1$ and $n q-m p-1 \in 2 \mathbb{N}$.

Example 1. $p=4, q=7, m=3$ and $n=9$. Clearly, $X=27, Y=25$ and $Z=37$.

\section{SOLUTIONS FAMILIES}

First of all, note that it is not easy to find integers $m, n, p, q$ such that $p q-m n=1$ and $n q-m p-1 \in 2 \mathbb{N}$.

The usage of matrices enables us to obtain a large number of solutions of (1.1). In this section, we will construct several infinite many families solutions of our triangular equation.

By completing squares in (2.1), we obtain

$$
\left(X+\frac{1}{2}\right)^{2}+\left(Y+\frac{1}{2}\right)^{2}=\left(Z+\frac{1}{2}\right)^{2}+\frac{1}{4} .
$$

Multiplying both sides by 4 , we get

$$
(2 X+1)^{2}+(2 Y+1)^{2}=(2 Z+1)^{2}+1 .
$$

By putting, $x=2 X+1, y=2 Y+1$ and $z=2 Z+1$, we obtain

$$
x^{2}+y^{2}=1+z^{2} \text {. }
$$

Since the proof of the following proposition can be seen easily we omit it.

Proposition 1. The two families $\left(\begin{array}{l}1 \\ a \\ a\end{array}\right),\left(\begin{array}{l}a \\ 1 \\ a\end{array}\right)$ are solutions of (3.1) for all integer $a$.

Theorem 2. There exist some $3 \times 3$ matrices $\mathfrak{M}$, with elements in $\{1,2,3\}$, such that if $\left(\begin{array}{l}x_{0} \\ y_{0} \\ z_{0}\end{array}\right)$ is a solution of (3.1) then $\mathfrak{M}\left(\begin{array}{l}x_{0} \\ y_{0} \\ z_{0}\end{array}\right)$ is also a solution.

Proof. Let $\mathfrak{M}=\left(\begin{array}{ccc}A & B & C \\ E & F & G \\ L & M & N\end{array}\right)$ and let $\left(\begin{array}{c}x_{0} \\ y_{0} \\ z_{0}\end{array}\right)$ a solution of (3.1).

It is clear that $\left(\begin{array}{ccc}A & B & C \\ E & F & G \\ L & M & N\end{array}\right)\left(\begin{array}{c}x_{0} \\ y_{0} \\ z_{0}\end{array}\right)=\left(\begin{array}{c}A x_{0}+B y_{0}+C z_{0} \\ F y_{0}+G z_{0}+E x_{0} \\ L x_{0}+M y_{0}+N z_{0}\end{array}\right)$.

Now, if the following system holds

$$
\begin{aligned}
A^{2}-L^{2}+E^{2} & =1 \\
B^{2}+F^{2}-M^{2} & =1 \\
C^{2}+G^{2}-N^{2} & =-1
\end{aligned}
$$




$$
\begin{aligned}
A B-L M+E F & =0 \\
A C-L N+E G & =0 \\
B C-M N+F G & =0
\end{aligned}
$$

then

$$
\left(A x_{0}+B y_{0}+C z_{0}\right)^{2}+\left(F y_{0}+G z_{0}+E x_{0}\right)^{2}-\left(L x_{0}+M y_{0}+N z_{0}\right)^{2}=1 .
$$

It follows from this, $\left(\begin{array}{c}A x_{0}+B y_{0}+C z_{0} \\ F y_{0}+G z_{0}+E x_{0} \\ L x_{0}+M y_{0}+N z_{0}\end{array}\right)$ is a solution of (3.1).

We will explore, in five steps, all solutions of the system $(S)$ are in the space $\{1,2,3\}^{9}$.

Case 1: $A=L=E=1$.

$$
\begin{aligned}
B^{2}+F^{2}-M^{2} & =1 \\
C^{2}+G^{2}-N^{2} & =-1 \\
B+F & =M \\
C+G & =N \\
B C-M N+F G & =0 .
\end{aligned}
$$

From (3.4), we obtain $F=1$ or $B=1$, then (3.3) implies $B=M$ or $F=M$ then $F=0$ or $B=0$ which is impossible.

Case 2: $A=1, L=E=2$.

$$
\begin{aligned}
B^{2}+F^{2}-M^{2} & =1 \\
C^{2}+G^{2}-N^{2} & =-1 \\
B-2 M+2 F & =0 \\
C-2 N+2 G & =0 \\
B C-M N+F G & =0 .
\end{aligned}
$$

Equations (3.5) and (3.6) imply that $B$ and $C$ are even.

Then from

$$
\begin{aligned}
M^{2}-F^{2} & =3 \\
N^{2}-G^{2} & =5 \\
M-F & =1 \\
N-G & =1 \\
M N-F G & =4 .
\end{aligned}
$$

Equation (3.7) gives $M=2, F=1$ and (3.8) gives $N=3, G=2$, 
then $\mathfrak{M}_{1}=\left(\begin{array}{ccc}A & B & C \\ E & F & G \\ L & M & N\end{array}\right)=\left(\begin{array}{lll}1 & 2 & 2 \\ 2 & 1 & 2 \\ 2 & 2 & 3\end{array}\right)$.

Case 3: $A=1, L=E=3$.

$$
\begin{aligned}
B^{2}+F^{2}-M^{2} & =1 \\
C^{2}+G^{2}-N^{2} & =-1 \\
B-3 M+3 F & =0 \\
C-3 N+3 G & =0 \\
B C-M N+F G & =0 .
\end{aligned}
$$

Equations (3.9) and (3.10) give us $B=C=3$, then

$$
\begin{aligned}
F^{2}-M^{2} & =-8 \\
G^{2}-N^{2} & =-10 \\
-M+F & =-1 \\
-N+G & =-1 \\
-M N+F G & =-9 .
\end{aligned}
$$

It is easy to see that equation (3.11) is impossible in $\{1,2,3\}^{2}$

Case 4 : $A=2$.

Equation 3.2 implies that $L=2, E=1$.

So, we get

$$
\begin{aligned}
B^{2}+F^{2}-M^{2} & =1 \\
C^{2}+G^{2}-N^{2} & =-1 \\
2 B-2 M+F & =0 \\
2 C-2 N+G & =0 \\
B C-M N+F G & =0 .
\end{aligned}
$$

Equations (3.12) and (3.13) give us $F=G=2$, thus

$$
\begin{aligned}
M^{2}-B^{2} & =3 \\
N^{2}-C^{2} & =5 \\
M-B & =1 \\
N-C & =1 \\
B C-M N+4 & =0 .
\end{aligned}
$$

Equation (3.14) gives us $M=2, B=1$ and (3.15) gives $N=3, C=2$. 
We obtain $\mathfrak{M}_{2}=\left(\begin{array}{ccc}A & B & C \\ E & F & G \\ L & M & N\end{array}\right)=\left(\begin{array}{lll}2 & 1 & 2 \\ 1 & 2 & 2 \\ 2 & 2 & 3\end{array}\right)$.

Case 5 : $A=3$.

Equation $\left(1_{0}\right)$ in $(S)$ implies that : $L=3, E=1$. Now

$$
\begin{aligned}
B^{2}+F^{2}-M^{2} & =1 \\
C^{2}+G^{2}-N^{2} & =-1 \\
3 B-3 M+F & =0 \\
3 C-3 N+G & =0 \\
B C-M N+F G & =0 .
\end{aligned}
$$

Equations (3.16) and (3.17) give us $F=G=3$, thus

$$
\begin{aligned}
M^{2}-B^{2} & =8 \\
N^{2}-C^{2} & =10 \\
B-M+1 & =0 \\
C-N+1 & =0 \\
B C-M N+9 & =0 .
\end{aligned}
$$

It is easy to see that equation (3.18) is impossible in $\{1,2,3\}^{2}$.

Corollary 1. The equation (1.1) admits infinite many solutions.

Proof. Let $\mathfrak{M}=\mathfrak{M}_{1}$ and $\left(\begin{array}{l}x_{0} \\ y_{0} \\ z_{0}\end{array}\right)=\left(\begin{array}{l}1 \\ 1 \\ 1\end{array}\right)$ a solution of (3.1) then $\mathfrak{M}^{n}\left(\begin{array}{l}1 \\ 1 \\ 1\end{array}\right)$ is a solution of (3.1) for all integer $n \geq 0$.

\section{NEW INTEGER SEQUENCES}

Using the on-line Encyclopedia of integer sequences (see [6]), we can easily check that the following two integer sequences formed by solutions $X$ of triangular equation (1.1) : 1, 5, 35, 203, 1179, 6929, 40391, 235415, 1372105, 7997213 $\ldots$ and $0,8,54,322$, $1884,10988,64050,373318,2175864,12681872, \ldots$ are new.

\subsection{Constructions}

1: We start with $\left(\begin{array}{l}3 \\ 1 \\ 3\end{array}\right)$, a solution of (3.1), thanks to theorem 2, by multiplying recursively by $\mathfrak{M}_{1}$, we obtain 


$$
\begin{aligned}
& \left(\begin{array}{l}
11 \\
13 \\
17
\end{array}\right),\left(\begin{array}{l}
71 \\
69 \\
99
\end{array}\right),\left(\begin{array}{l}
407 \\
409 \\
577
\end{array}\right),\left(\begin{array}{l}
2379 \\
2377 \\
3363
\end{array}\right),\left(\begin{array}{l}
13859 \\
13861 \\
19601
\end{array}\right),\left(\begin{array}{c}
80783 \\
80781 \\
114243
\end{array}\right), \\
& \left(\begin{array}{l}
470831 \\
470833 \\
665857
\end{array}\right),\left(\begin{array}{l}
2744211 \\
2744209 \\
3880899
\end{array}\right),\left(\begin{array}{l}
15994427 \\
15994429 \\
22619537
\end{array}\right), \ldots \text { are also solutions of }(3.1) .
\end{aligned}
$$

We get, $x=3,11,71,407,2379,13859,80783,470831,2744211,15994427, \ldots$

But $x=2 X+1$, so

$$
X=1,5,35,203,1179,6929,40391,235415,1372105,7997213, \ldots
$$

is a new sequence of odd integers formed by solutions $X$ of our triangular equation (1.1).

2: We start with $\left(\begin{array}{l}1 \\ 5 \\ 5\end{array}\right)$ a solution of (3.1), thanks to theorem 2, by multiplying recursively by $\mathfrak{M}_{2}$, we obtain

$$
\begin{aligned}
& \left(\begin{array}{l}
17 \\
21 \\
27
\end{array}\right),\left(\begin{array}{l}
109 \\
113 \\
157
\end{array}\right),\left(\begin{array}{l}
645 \\
649 \\
915
\end{array}\right),\left(\begin{array}{l}
3769 \\
3773 \\
5333
\end{array}\right),\left(\begin{array}{l}
21977 \\
21981 \\
31083
\end{array}\right),\left(\begin{array}{l}
128101 \\
128105 \\
181165
\end{array}\right), \\
& \left(\begin{array}{c}
746637 \\
746641 \\
1055907
\end{array}\right),\left(\begin{array}{l}
4351729 \\
4351733 \\
6154277
\end{array}\right),\left(\begin{array}{l}
25363745 \\
25363749 \\
35869755
\end{array}\right), \ldots \text { are also solutions of }(3.1) .
\end{aligned}
$$

We get, $x=1,17,109,645,3769,21977,128101,746637,4351729,25363745, \ldots$

But $x=2 X+1$, then

$$
X=0,8,54,322,1884,10988,64050,373318,2175864,12681872, \ldots
$$

is a new sequence of even integers formed by solutions $X$ of our triangular equation (1.1).

\section{REFERENCES}

[1] M. A. Bennett, "A question of Sierpinski on triangular numbers.” Integers, vol. 5, no. 1, pp. A25, 2, 2005.

[2] J. A. Ewell, "On sums of three triangular numbers." Fibonacci Quart., vol. 26, no. 4, pp. 332-335, 1988.

[3] I. Joshua and J. Lenny, "Arithmetic progressions involving triangular numbers and squares." $J$. Comb. Number Theory, vol. 5, no. 3, pp. 165-179, 2013.

[4] R. Keskin and O. Karaatli, "Some new properties of balancing numbers and square triangular numbers." J. Integer Seq., vol. 15, no. 1, pp. Article 12.1.4, 13, 2012.

[5] W. Sierpinski, "Theorem on triangular numbers." Elem. Math., vol. 23, pp. 31-32, 1968.

[6] N. Sloane, The On-Line Encyclopedia of Integer Sequences., https://oeis.org/. 
Authors' addresses

\section{Abdelkader Hamtat}

A.T.N. Laboratory, Faculty of Mathematics, USTHB BP 32, El Alia, 16111, Bab Ezzouar, Algiers ALGERIA

E-mail address: ahamttat@gmail.com

Djilali Behloul

A.T.N. Laboratory, Faculty of Mathematics, USTHB BP 32, El Alia, 16111, Bab Ezzouar, Algiers ALGERIA

E-mail address: dbehloul@yahoo.fr 